\title{
Roles of Brain Criticality and Multiscale Oscillations in Temporal Predictions for Sensorimotor Processing
}

\section{Palva, Satu}

2018-10

Palva , S \& Palva , J M 2018 , ' Roles of Brain Criticality and Multiscale Oscillations in

Temporal Predictions for Sensorimotor Processing ', Trends in Neurosciences, vol. 41 , no.

10 , pp. 729-743 . https://doi.org/10.1016/j.tins.2018.08.008

http://hdl.handle.net/10138/305688

https://doi.org/10.1016/j.tins.2018.08.008

publishedVersion

Downloaded from Helda, University of Helsinki institutional repository.

This is an electronic reprint of the original article.

This reprint may differ from the original in pagination and typographic detail.

Please cite the original version. 


\title{
Roles of Brain Criticality and Multiscale Oscillations in Temporal Predictions for Sensorimotor Processing
}

\author{
Satu Palva ${ }^{1, \star}$ and J. Matias Palva ${ }^{1, \star}$
}

Sensorimotor predictions are essential for adaptive behavior. In natural environments, events that demand sensorimotor predictions unfold across many timescales, and corresponding temporal predictions (either explicit or implicit) should therefore emerge in brain dynamics. Neuronal oscillations are scalespecific processes found in several frequency bands. They underlie periodicity in sensorimotor processing and can represent temporal predictions via their phase dynamics. These processes build upon endogenous neural rhythmicity and adapt in response to exogenous timing demands. While much of the research on periodicity in neural processing has focused on subsecond oscillations, these fast-scale rhythms are in fact paralleled by critical-like, scale-free dynamics and fluctuations of brain activity at various timescales, ranging from seconds to hundreds of seconds. In this review, we put forth a framework positing that critical brain dynamics are essential for the role of neuronal oscillations in timing and that cross-frequency coupling flexibly organizes neuronal processing across multiple frequencies.

\section{Critical-like Multiscale Brain Oscillations for Processing a Critical-like Multiscale Environment?}

Perception and action in natural environments necessitate interplay among representations of past events, the current state, as well as predictions of both future events and the consequences of our own actions across a continuum of vastly different timescales. Notably, natural visual [1] and auditory [2] scenes are characteristically scale-free and arrhythmic signals with power-law scaling behavior (Box 1 and Figure $1 \mathrm{~A}-\mathrm{C}$ ). Such lack of scaling or periodicities is perhaps not too surprising, considering that many aspects of our environment are ongoing processes involving nonlinear interactions among many agents, or are products of such processes, and thereby encompass complex or 'critical' spatiotemporal dynamics [3]. Critical dynamics appear in systems poised at a transition between two phases, and such systems are characterized by stochastic fractal-like architectures, power-law correlations, and rapid intermittent state transitions $[3,4]$. However, in many real-world settings scale-free environmental contexts are accompanied by distinct scale-specific phenomena (i.e., periodic or quasiperiodic signals; Box 1). Such scale-specific components arise both from artificial sources and from biological systems, for instance through phenomena with rhythmic components, such as gait and production of speech and music (Figure 1C-E).

What are the neural processes that facilitate effective perception, cognition, and action in environments that consist of both scale-free and scale-specific temporal structures? The range of behaviorally relevant environmental temporal scales is well paralleled by the spectrum of
Highlights

Neuronal oscillations in ongoing brain activity adapt to behaviorally relevant sensorimotor rhythmicities by phase prediction and entrainment.

Neuronal oscillations also endogenously impose rhythmicity on perceptual, attentional, and actiongeneration processes.

These oscillations are spectrally distributed and functionally specialized, and thus they depend on cross-frequency coupling for functional integration.

Infraslow $(0.01-0.1 \mathrm{~Hz})$ and slow $(0.1-$ $1 \mathrm{~Hz}) \mathrm{EEG}$ and BOLD fluctuations are phase coupled with $>1 \mathrm{~Hz}$ neuronal oscillations and slow behavioral performance fluctuations.

Neuronal oscillations are likely to operate in a critical-like state with significant interindividual variability, which has fundamental implications for the capability of individual subjects' neuronal networks to synchronize, respond to phase-resetting stimuli, and entrain to exogenous oscillation.

${ }^{1}$ Neuroscience Center, Helsinki Institute of Life Science, University of Helsinki, Haartmaninkatu 3, 00029 Helsinki, Finland

${ }^{*}$ Correspondence:

satu.palva@helsinki.fi (S. Palva) and matias.palva@helsinki.fi (J.M. Palva). 
Box 1. Arrhythmic, Quasiperiodic, and Periodic Signals Characterize Natural Environments and Brain Activity

The spatiotemporal structure of environmental information is statistically fractal-like and typically lacks characteristic scales (i.e., it is 'scale-free'). For example, pictures [1] and audio recordings [2] of natural (including urban) environments (Figure $1 \mathrm{~A}$ ) have $1 / \mathrm{f}^{\mathrm{a}}$-noise like power spectra with roughly a log-log linear decay of power as a function of frequency (Figure 1B) and a lack of peaks both in single trials and in data averaged across many trials. However, especially in human-made contexts or in the presence of other animals/humans, it is easily conceivable to have behaviorally relevant information that has a characteristic scale (i.e., that is at least transiently oscillatory; Figure $1 \mathrm{C}$ ). Processes that produce recurrent patterns with an irregular period are termed 'quasiperiodic' oscillations. In single trials, quasiperiodic processes may exhibit power-spectral peaks (Figure 1D), but with adequate variability or summation of signals from many processes expressing distinct periodicities in different trials, the averaged power spectrum may be experimentally indistinguishable from the spectra of genuinely arrhythmic signals (Figure 1B). In the case of oscillations that have stable periodicity across many trials, both the single trial and averaged power spectra have peaks. The fundamental difference between arrhythmic and quasiperiodic processes is that for quasiperiodic, exactly like for periodic processes, phase is a functionally significant variable whereas for arrhythmic processes, phase is irrelevant and poorly defined because no periodicities exist.

Human psychophysical performance is also characterized by scale-free dynamics. Concurrent EEG recordings reveal infraslow $(0.01-0.1 \mathrm{~Hz})$ neuronal fluctuations of which the phase is correlated with the fluctuations of behavioral performance [101]. This implies that infraslow EEG fluctuations are quasiperiodic oscillations because their averaged power spectrum does not exhibit salient peaks (Figure 1G) such as the one observed at $10 \mathrm{~Hz}$ for alpha oscillations, even if peaks may be observed in shorter data segments [120]. Infraslow scalp EEG fluctuations are coupled with fMRI resting-state networks [106] and currently represent the slowest process where phase biases behavior.

neuronal activities, spanning from frequencies as low as $0.01 \mathrm{~Hz}$ up to $200 \mathrm{~Hz}$. Neuronal oscillations are scale-specific rhythmic or periodic excitability fluctuations in neuronal populations that also modulate psychophysical performance (Figure 1F,G). They impose an oscillation-phase-dependent bias on neuronal firing [5], so that firing is facilitated in the highexcitability phase and suppressed in the low-excitability phase. Oscillation phase progression intrinsically contains prospective temporal information about the moments when neuronal processing will be enhanced. Hence oscillations, by definition, imply a mechanism for representing temporal predictions. It appears, indeed, that the brain uses oscillations to sample sensory information and motor actions rhythmically, and that it adaptively exploits the oscillation phase to optimize processing in environmentally appropriate moments.

In this review, we aim to bridge three lines of research relevant to this theme. First, we recapitulate the often-suggested role of neuronal oscillations in providing endogenous and adaptive clocking for momentarily enhancing neuronal processing. Second, we propose that distinct forms of cross-frequency coupling play functional roles in organizing the cooperation of neuronal processes manifesting in distinct timescales or frequency bands. Third, we suggest that critical-like dynamics, as implied in the slow fluctuations of these oscillations, are essential for enabling their functional roles by endowing them with three crucial properties: rapid phase resetting, entrainability, and synchronizability.

\section{Adaptation of Neuronal Oscillations to External Rhythmicity via Entrainment}

Efficient neuronal processing requires adaptive optimization to match the expected temporal structures in sensory information at various timescales. In paradigms involving periodic stimulus presentation, this temporal structure is salient and, indeed, such periodic stimuli often entrain neuronal oscillations at the corresponding frequency. For example, auditory stimuli presented at delta frequencies $(1-4 \mathrm{~Hz})$ entrain delta oscillations and lead to improved stimulus detection probabilities compared to nonperiodic stimulus presentation [6-10]. Moreover, if there is temporal variability in the acoustic sequences, the phase relationship of delta oscillations in the auditory cortex and the stimulus sequence predicts the detection of near-threshold stimuli 
(A)

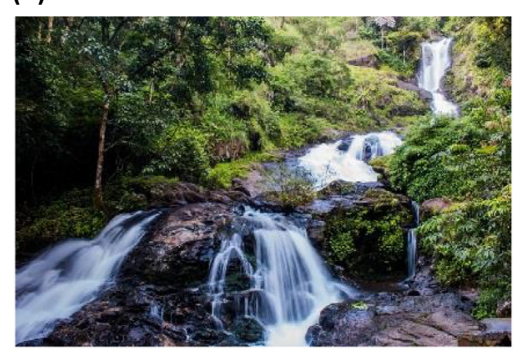

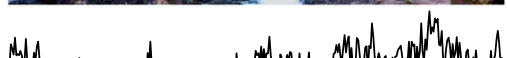

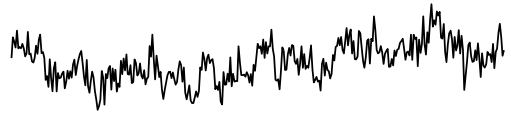

(B)
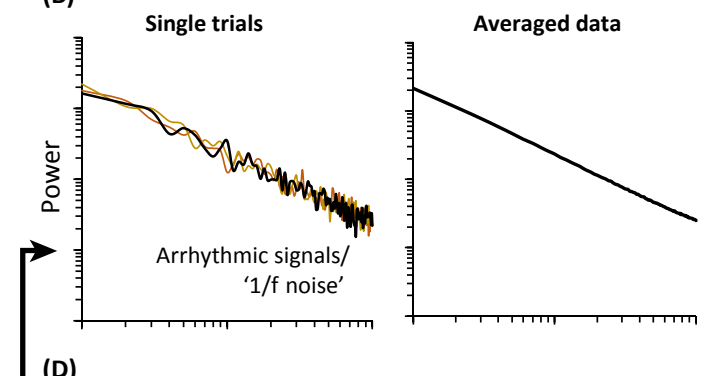

(D)

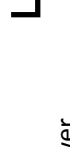
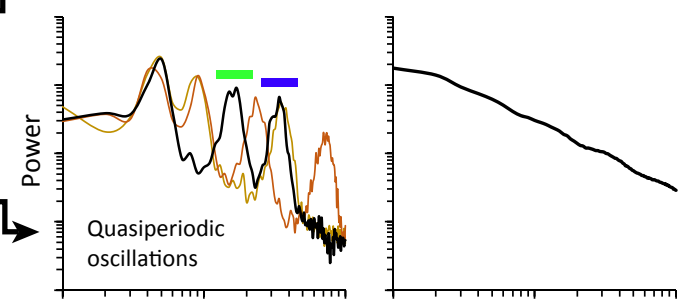

(E)
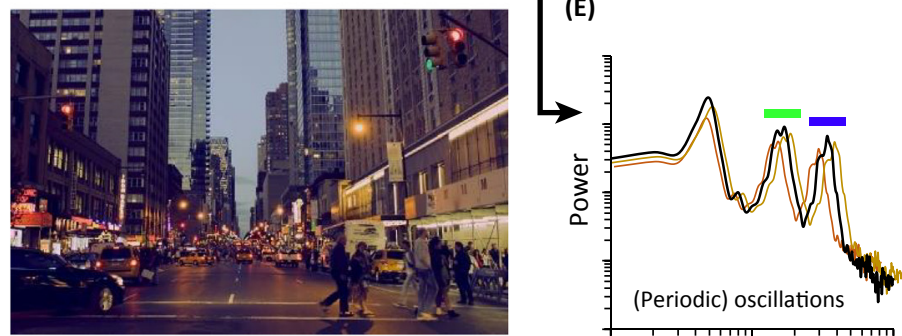

Frequency

(G)

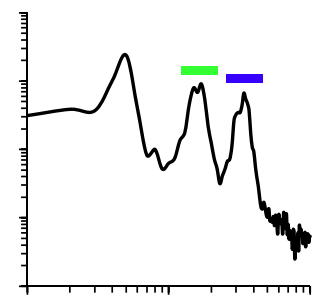

Frequency
(F)

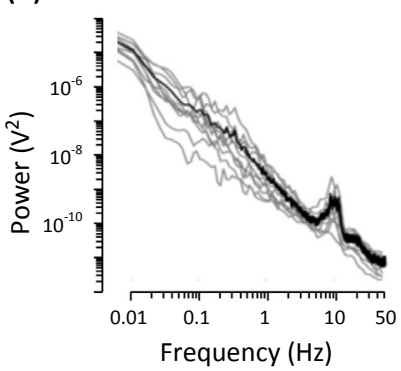

Trends in Neurosciences

Figure 1. Arrhythmic, Quasiperiodic, and Periodic Signals in the Environment and in the Brain. (A) Natural images and audio recordings typically exhibit power-law statistics. The signal trace is a simulated $1 / f$ noise that $(B)$ in both single realizations (left) and in averaged data (right) yields $1 / f$ power spectra. (C) Signals emerging from both natural environments and in ones shaped by humans often contain periodicities. In the image shown here, periodicities could be related, for instance, to police car sirens, traffic lights, and ringtones, as well as human gait, speech, and music. Mixtures of transient periodicities from multiple sources or quasiperiodic oscillators with a large variability in oscillation periods give rise to power spectra (D) that exhibit peaks at the corresponding frequencies (green and blue bars) in single trials, even though in averaged data the spectra may be void of peaks. (E) Only oscillations with a stable period retain peaks in averaged power spectra. (F) Human psychophysical performance is often characterized by scale-free dynamics. In a somatosensory threshold stimulus detection task, for instance, detected (blue) and undetected (red) stimuli are clustered with power-law, long-range, temporal correlations [101,103] (here, data are from a representative subject). Further, scalp EEG recordings reveal infraslow $(0.01-0.1 \mathrm{~Hz})$ neuronal fluctuations (black) of which the phase (green) is predictive of behavioral timing in 10-100 s timescales. Infraslow fluctuations (black) are obtained by bandpass filtering the raw EEG signal (0-200 Hz, grey)

(Figure legend continued on the bottom of the next page.) 
(A)

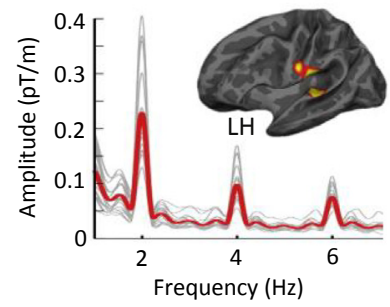

(B)

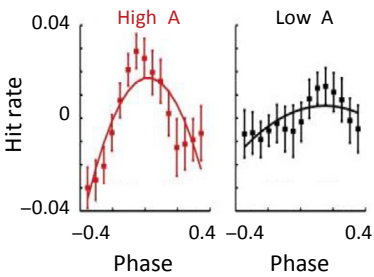

(C)

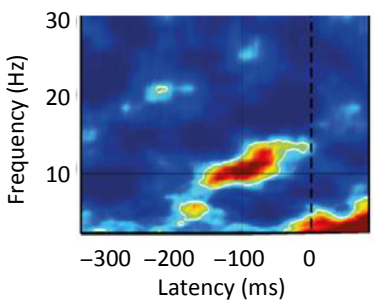

(D)
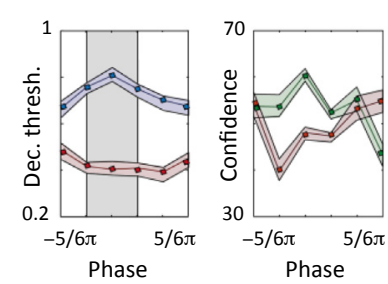

(E)

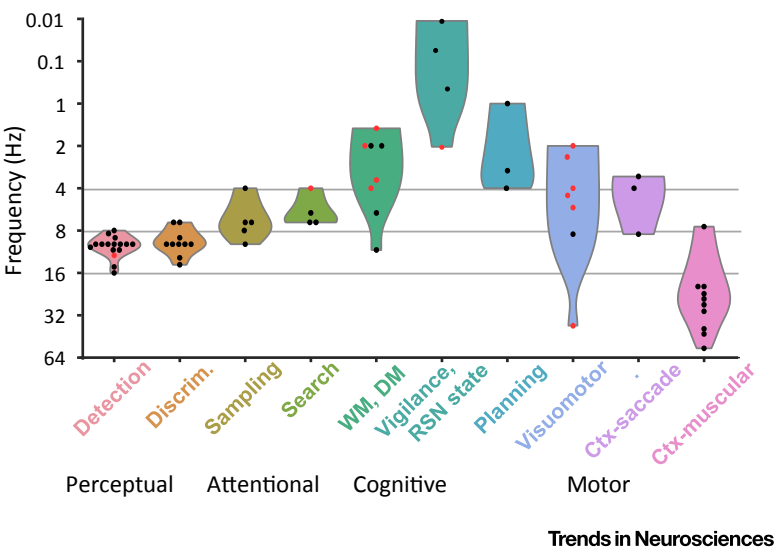

Trends in Neurosciences

Figure 2. Both Entrained and Intrinsically Periodic Sampling Support Perception and Attentional Functions. (A) Temporally variable auditory stimulus sequence of an average rhythm of $2 \mathrm{~Hz}$ entrains $2-\mathrm{Hz}$ delta oscillations in the auditory cortex. (B) Neuronal 2- $\mathrm{Hz}$ oscillations express temporal expectations so that the hit rate is dependent on the oscillation phase, and it is more so at high (left) oscillation amplitude, and much less so at low (right) amplitudes. Adapted from [6]. (C) Prestimulus phase locking of alpha oscillations in a visual detection task that manipulated perceptual expectations and attention (yellowred colors indicate $p<0.001)$. (D) Left: occipital alpha phase predicted the weighting of expectations on decision threshold (threshold for reporting stimulus presence; blue, 'expect stimulus presence'; red, 'expect stimulus absence'; decision threshold [Dec. thresh.]; blue $25 \%$, and red $75 \%$ ). Right: alpha phase also reflected the influence of expectations on confidence (green, 'congruent' [stimulus present/absent when expecting presence/absence] red, 'incongruent' (vice versa)). Adapted from [26]. (E) Illustration of the frequencies observed in studies finding a correlation between behavioral/action-related measures (horizontal axis) and the phase of spontaneous (black dots) or entrained (red dots) neuronal oscillations. WM, working memory; DM, decision making; RSN, resting-state network; Ctx, cortex. This collection of studies is tabulated in Table $\mathbf{S} 1$ in the supplemental information online and includes a subset of studies earlier reported in [19].

in a manner dependent on the delta amplitude [6] (Figure 2A,B). Thus, successful entrainment of neuronal oscillations is paralleled by a behavioral advantage.

Spoken language is of special importance in human social systems. Unlike periodically presented auditory stimuli, the speech modulation spectrum spans a wide range of frequencies between $0.25 \mathrm{~Hz}$ and $30 \mathrm{~Hz}$ [11]. Correspondingly, speech stimuli entrain brain rhythms similarly in multiple frequency bands [12-15]. Interestingly, however, speech modulation exhibits a mode at around the same frequency of $5 \mathrm{~Hz}$ for many languages. A recent study using electrical brain stimulation with speech envelopes showed that enhancing neuronal entrainment leads to improved speech comprehension with the effect peaking at $5 \mathrm{~Hz}$ [16]. Entrainment is not limited though to auditory stimuli. Natural-like visual stimuli $[17,18]$ also entrain oscillations in multiple frequencies. In the audiovisual domain, concurrent phase maxpixel.net. (F) and (G) adapted from [101]. 
entrainment of delta- (1-4 Hz) and theta-frequency $(5-8 \mathrm{~Hz})$ band oscillations in the auditory cortex has been proposed to provide a basis for continuous tracking of naturalistic audiovisual streams and integration of auditory and visual information [10]. The phase dynamics of neuronal oscillations thus appear both highly adaptive to temporal predictability in the environment and actively utilized in the timing of neuronal processing to optimize task performance.

\section{Periodic Perceptual and Attentional Sampling of Sensory Information}

Apart from oscillatory entrainment by external stimuli, a considerable body of research has revealed how perception is also endogenously dependent on prestimulus neuronal oscillations [19], so that sensory processing is facilitated when the stimulus presentation evokes activity in the high-excitability phase of neuronal oscillations [5,20]. Behavioral data, as well as electrophysiological evidence obtained using electroencephalography (EEG) and magnetoencephalography (MEG), suggest that visual perception is intrinsically sampled rhythmically in the alpha-frequency band (and possibly other bands as well). Primary evidence for this comes from findings showing that the prestimulus phase [21-25] of alpha oscillations modulates the detection and discrimination of visual stimuli. A recent study dissecting the putative functional roles of the prestimulus phase effects examined how the prestimulus occipital alpha phase relates to perceptual decisions. The study showed that the prestimulus occipital alpha phase predicts, specifically, the biasing of the decision threshold by expectations and the influence of confidence in a manner independent of attentional modulation [26] (Figure 2C,D). Further evidence for discretized or rhythmic alpha-band perception is provided by findings that, within alpha cycles, visual object information is fused [27-29], suggesting that alpha cycles reflect the smallest temporal units of subjective visual perception. Most studies on the topic have observed such intrinsic perceptual sampling to take place at $\sim 10 \mathrm{~Hz}$ (Figure 2E). Interestingly, attentional and other top-down temporal predictions also appear to impose rhythmicity on the sampling of sensory information [21,26,30-32]. For attended sampling, however, perception is often predicted by low alpha- or theta-band phases (Figure 2E). Thus, attention-mediated sampling appears to operate at lower frequencies than those associated with perceptual sampling. Moreover, if two sensory stimuli are observed concurrently, perception is sampled at delta frequencies [6,32-34], as if a central theta-frequency attentional sampler would focus cycle-by-cycle on alternating object locations [19,32]. The alpha-vs.-theta dichotomy of perceptual-vs.-attentional sampling periodicity is further underscored by the associated scalp-EEG topographies of these effects, which are occipital for alpha and frontal for theta, respectively [19]. Emphasizing the importance of experimental nuances, in an attentive temporal cueing task, perceptual accuracy was still determined by the phase of posterior alpha rather than that of frontal theta [35]. Importantly, this study also showed that the pretarget stimulus alpha phase was influenced by the preceding cue, which implies that accurate temporal predictions may be implemented by top-down control of the timing of visual cortex alpha oscillations [35]. Putatively, such top-down control could be achieved through frontovisual alpha-band phase synchronization that link frontal and visual cortices during visuospatial attention [36]. These frequency differences among perceptual and attentional sampling suggest that delta, theta, and alpha oscillations form temporal hierarchies matching the hierarchies of cognitive operations so that higher level functions are associated with slower frequencies.

Modulation of perception by intrinsic brain rhythms in sensory modalities other than vision has been less studied. In the somatosensory modality, both alpha and beta oscillations have been associated with modulations of detection probability $[37,38]$. Also in the auditory domain, target detection probability has been shown to depend on both theta- and alpha-band prestimulus phases $[39,40]$. These studies thus suggest that endogenous temporal integration and parsing 
of sensory information could have a shared mechanistic basis across sensory modalities, but with distinctions in entrainability and the role of the prestimulus phase [41].

Brain stimulation studies provide a complementary line of evidence for assessing the relationship of perception and the prestimulus phase of neuronal oscillations. Single-pulse transcranial magnetic stimulation (TMS) applied over the human visual cortex induces illusory perceptions. Similarly to perceptions induced by visual stimuli, TMS-induced perceptions are most reliably triggered in a given phase of prestimulus alpha oscillations $[42,43]$. Providing causal evidence for the relationship between alpha phase and perception in the auditory modality, alphafrequency-modulated transcranial direct current stimulation (tDCS) biases the stimulusdetection threshold in a phase-dependent manner [44]. Hence, in both auditory and visual modalities, brain stimulation studies support the idea of alpha-frequency perceptual sampling, but, to our knowledge, there are no brain stimulation studies testing the causality of this phase relationship by specifically manipulating the phase of prestimulus neuronal oscillations.

\section{Sensorimotor Coordination by Brain Rhythmicity}

In natural environments, action and perception are invariably intertwined, and perception is always 'active' in the sense of involving cognitive and motor control of sensory sampling. In primates, the sampling of visual information is dictated by eye movements, especially by saccades and microsaccades that typically take place at a rate of one to three discrete movements per second [45]. Saccades sample visual information according to both visual scene requirements and task attentional demands [46,47]. The apparent delta-band rhythmicity of saccades has been suggested to arise so that each saccade is initiated at the end of a refractory period, when the processing of the previous saccade is finished [48]. Nonetheless, saccades have been shown to be phase-locked to prestimulus alpha oscillations in the visual and medial temporal cortices during memorization of natural images, with the strength of phase-locking predicting memory performance [49]. Importantly, as in intrinsic perceptual/ attentional sampling, the phase of presaccade theta oscillations per se predicts the perceptual magnitude of perisaccadic mislocalization [50], and the phase of alpha oscillations (saccadic reaction times) [22]. Saccades are followed by precisely timed synchronous discharges [51] and pronounced changes in oscillatory activity in the visual system [46,52,53]. As a result, each fixation onset is rapidly followed by enhanced neuronal responsiveness and phase alignment among neuronal oscillations, especially in the theta band, as shown for instance in V1 and in the hippocampus [52,54]. Saccades thus phase-reset cortical oscillations to endow the new retinal input with the oscillation phase, yielding optimal excitability. Regardless of the precise mechanism generating saccade periodicity, saccades thus act in many ways as an intrinsic counterpart to the delta-frequency stimulus entrainment discussed above.

Saccades also appear to impact the coherence of neuronal oscillations among brain regions, and thereby, possibly, the communication between them. Specifically, interareal coherence of alpha oscillations has been found to link cortical areas that encode the retinal location of a visual stimulus before and after a saccade, which suggests that these alpha oscillations support the construction of stable visual space representations around the time of saccades and the resulting shifts in retinal image location [53]. In sum, it appears that the rhythmic perceptual and attentional sampling is achieved via multiplexing of delta-band eye movements and cortical oscillations with theta and alpha oscillations, both of which influence saccade timing and become phase aligned after the saccade.

Alpha oscillations, and their functional roles, have been the topic of many theories and discussions. Recent years have seen a revived interest in questions relating to the neuronal mechanisms underlying alpha oscillations. Neuronal firing is modulated by the alpha phase [49]; 
and the presence of alpha-oscillation current generators in all cortical layers in primary sensory areas suggests that alpha oscillations are involved in both feedforward and feedback processes $[50,55,56]$, in line with the idea that alpha oscillations may regulate both intrinsic sensory processing and its attentional sampling. The contribution of alpha phase synchronization is exemplified by studies examining the roles of frontal areas in attention and visual perception. In the primate frontal eye fields (FEFs), saccade-related activity peaks around the time of saccade initiation [57]. Synchronization and phase-coherence between FEF and visual cortices is associated with attentional modulation of neuronal activity [58,59]. During visual attention, cortical processing in the visual areas is modulated by thalamic input through alpha-band synchronization [60], suggesting that thalamic alpha oscillations may contribute to cortical alpha-band rhythmicity in attentive states. Studies using TMS showed that perturbing FEF activity can 'break' the coupling between visuospatial attention and eye movements [61] and slow down perception [62]. In humans, the sources of attention-related synchronization in the prefrontal cortex are more widespread, but, overall, long-range alpha phase synchronization among frontal, parietal, and visual cortices seems to be salient, as seen for instance in a visual attention task [36]. Such links provide a putative network-level basis for how visual perception is dependent on the phase of local alpha oscillations in occipital areas.

The roles of rhythmic brain activity in action generation go beyond the oculomotor system. In fact, adaptive motor control involving cortical oscillations entrained by environmental periodicities seems to be fairly widespread. The speed of motor actions is dependent on delta-band phase in both human scalp EEG [8,63] and intracranial EEG [64], and motor responses are facilitated during the high-excitability phases, similarly to what is seen in perceptual sampling. Slow finger movements are discretized into alpha-frequency steps that are phase coupled with motor cortical alpha oscillations [65], supporting the idea that alpha-band oscillations underlie temporal framing of both sensory information and actions. Finally, coordination between the motor cortex and muscles is supported by corticospinal coherence, which (depending on experimental conditions) was shown to take place in the beta- or gamma-bands in humans [66-68], and in the beta-band in monkeys $[69,70]$. The motor cortex is also phase-synchronized with other brain regions in a task dependent manner. For example, concurrent synchronization in delta and gamma bands connects sensorimotor, frontal, and parietal brain areas during conscious somatosensory perception and action [71]. For speech perception, theta-band synchronization between auditory and speech-motor regions was observed while participants listened to syllables [72]. Direct evidence for the importance of neuronal oscillation phase in action coordination comes from studies where TMS was applied over the motor cortex. The TMS-evoked muscle activation was dependent on the phase of cortical slow [73] and beta-band [74] oscillations. Hence, discrete sampling and phase-dependent coding extend from perception and attention, as previously discussed, to motor control and action generation as well.

\section{Oscillations Contribute to Evidence Accumulation for Temporal Information Acquisition}

Many ecologically valid situations necessitate explicit estimation of the timing of events in both natural environments and in ones shaped by humans. Explicit representation of temporal information in the brain has been frequently investigated using interval timing tasks. In these tasks, subjects are asked to make judgments based on temporal intervals between pairs of events. Explicit estimation of time in intervals from seconds to minutes involves activity in many areas related to motor control such as in premotor and supplementary motor areas, basal ganglia, and the cerebellum, but also in the prefrontal cortex, frontal operculum, and posterior parietal cortex [75]. Theoretical models of time estimation suggest that the timing process is dependent on the accumulation of implicit duration information over the task-relevant time window [76-78]. It has 
been proposed that this accumulation of information could be achieved through rhythmic or periodic fluctuations (i.e. via neuronal oscillations) [79]. This idea is supported by findings showing that the subjective perception of passage of time is rhythmic [80]. Yet, data on the oscillatory mechanism underlying explicit time estimation are scarce. Temporal predictions in monkeys have been related to beta oscillations [81]. Similarly, in source-modelled human MEG data, timeestimation in a working memory (WM) task is correlated with the amplitude of beta oscillations in the sensorimotor (SM) areas and posterior parietal cortex (PPC) [82]. As beta oscillations are generally linked to motor coordination, such neuroanatomical localization supports the idea that the explicit estimation of temporal intervals is tightly linked to action generation. Dynamic associations and coupling between temporal estimations and actions would allow fast and accurate action generation according to environmental demands.

\section{Cross-frequency Interactions between Oscillations at Multiple Scales Govern Neuronal Processing across Cortical Hierarchies}

The studies reviewed above converge to show that phase dynamics of oscillations in multiple frequency bands, with distinct neuroanatomical sources and functional roles, are essential for perceptual and attentional sampling, sensorimotor coordination, and temporal predictions. The frequencies of neuronal oscillations underlying this temporal coordination and setting the temporal integration windows are dependent on the level of cognitive hierarchy (Figure 2E). Coordination and functional integration of such multiscale neuronal oscillations demands cross-frequency coupling (CFC) mechanisms such as cross-frequency phase synchronization (CFS) $[83,84]$ or phase-amplitude coupling (PAC) [85] (Figure 3A-C). Yet, whether and how these mechanisms coordinate neuronal processing, from rhythmically sampled perception, to periodically implemented steps in continuous actions, has remained incompletely understood.

PAC has been repeatedly observed between slow $(<1 \mathrm{~Hz})$ and fast $(>1 \mathrm{~Hz})$ oscillations (Figure 3D) as well as among fast oscillations. It has been suggested to provide a generic temporal segmentation mechanism for cognitive functions [86,87]. According to one view, for instance, sensory information is represented in gamma oscillations, which are then segmented by PAC into gamma bursts with slower oscillations $[88,89]$. In line with these ideas, gamma oscillation amplitudes are indeed coupled to the phase of delta, theta, and/or alpha oscillation phases during attentional sampling of sensory information, both in humans and non-human primates [7,9,90], as well as during working memory [91]. During entrainment of multiscale neuronal oscillations in speech perception, the amplitude of gamma oscillations in the auditory cortex is locked to the theta phase, which has been proposed to support speech segmentation $[92,93]$. Also the phase of a slow $\sim 1 \mathrm{~Hz}$ component that is locked to phrasal contents has been found to be coupled with the amplitude of beta-frequency oscillations in motor areas [14]. Observations of PAC during attention and speech perception tasks suggest that it plays a functional role in expressing temporal predictions by supporting time windows of enhanced excitability defined by the phase of delta, theta, and alpha oscillations.

Cross-frequency phase synchronization (CFS) is another form of CFC, distinct from PAC, and is characterized by a stable phase-difference between two neuronal assemblies oscillating with an $m: n$ frequency ratio $[83,94,95]$. Since, unlike in PAC, the phases of both the faster and slower oscillation are relevant for CFS, it necessarily operates at the temporal accuracy of the faster oscillation and endows the coupled assemblies with a consistent spike-timing relationship. CFS may thus trigger neuronal coincidence detection mechanisms and serve the regulation of neuronal communication similarly to 1:1 synchronization. CFS has been observed in human sensor-level MEG recordings during a WM intensive mental calculation task [83], during a WM [96] task, as well as during attention demanding visual perception [97]. More 
(A) Broadband $(10+20+80 \mathrm{~Hz})$

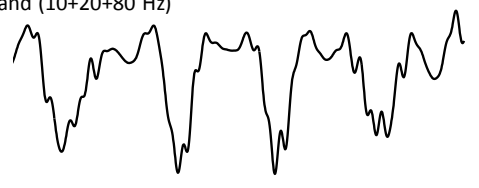

(B)

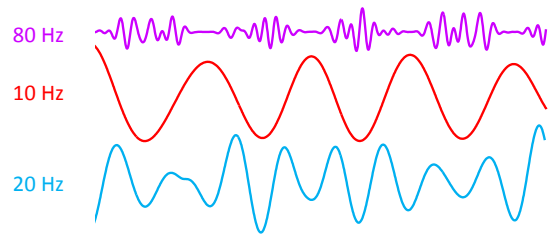

(C)

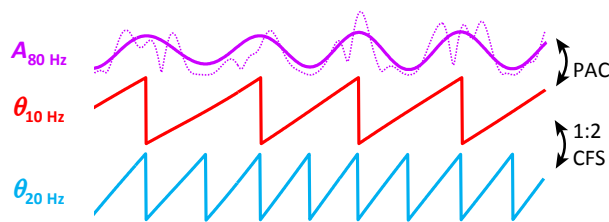

(F)


(D)

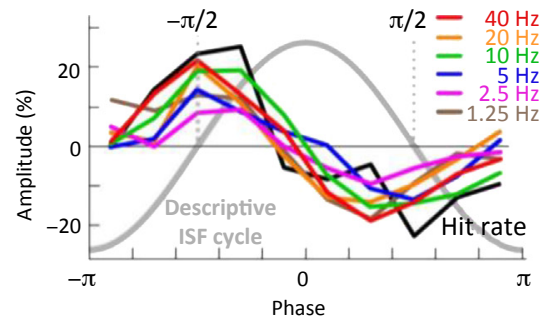

(E)

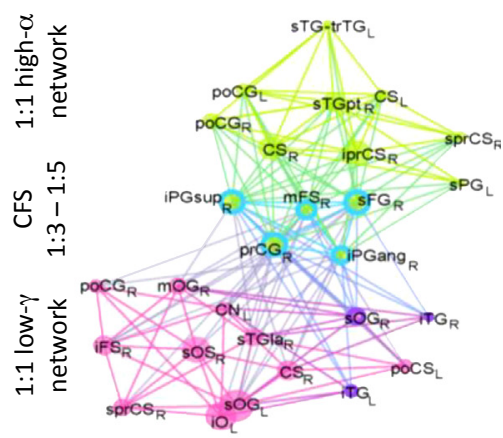

(G)

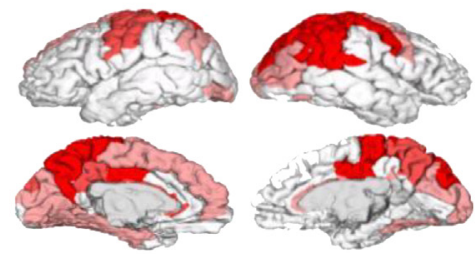

(H)

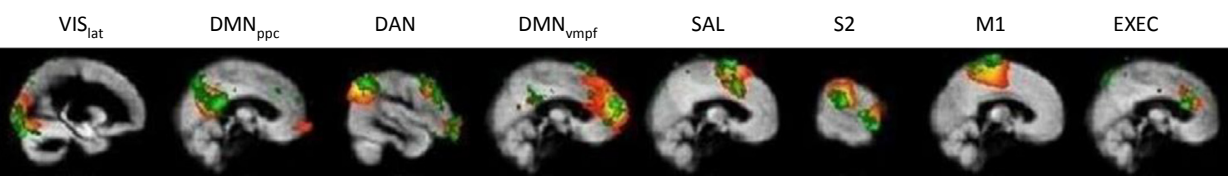

Trends in Neurosciences

Figure 3. Cross-frequency Interactions for Coupling near and far Frequencies. (A) Schematic of oscillatory cortical hierarchies with an example broadband signal composed of simulated oscillations in three frequency bands (B). Note that the seemingly nonsinusoidal and asymmetric appearance of the broadband signal is caused exclusively by the genuine 1:2 phase synchronization between the sinusoidal 10 and $20 \mathrm{~Hz}$ oscillations. (C) Schematic illustration of phase-amplitude coupling (PAC) and cross-frequency synchronization (CFS). PAC indicates that the amplitude envelope of the fast oscillation $\left(A_{80 \mathrm{~Hz}}\right.$, dotted line) is correlated with the phase of the slow oscillation $\left(\theta_{10} \mathrm{~Hz}\right)$. PAC may be estimated by filtering $A_{80 \mathrm{~Hz}}$ at the slow frequency (here $10 \mathrm{~Hz}$, filled line) and evaluating 1:1-phase coupling with the phase-locking value [120]. CFS indicates cross-frequency correlation between the phases of two signals at frequencies $f_{x}$ and $f_{y}$ that have a (usually small) integer ratio $n$ : $m$ so that $n f_{x}=m f_{y}$. Unlike PAC, CFS enables consistent spike-time relationships in the underlying neuronal populations. Here we illustrate 1:2 CFS of beta and alpha oscillations, which in human brain activity is by far the most prevalent [83,84]. (D) PAC among infraslow oscillations and $1-40 \mathrm{~Hz}$ oscillations characterizes scalp EEG in detection tasks, where the infraslow phase is also correlated with behavioral hit rate. Note that such PAC inherently also introduces cross-frequency amplitude-amplitude correlations among the 1-40 Hz oscillations. Adapted from [101]. (E) CFS during working memory retention links hubs of 1:1 within-frequency-synchronized networks and may serve cross-frequency functional integration (ang, angular; C, central; CN, cuneus; F, frontal; G, gyrus; i, inferior; L, left; la, lateral; m, middle; O, occipital; P, parietal; po, post; pr, pre; R, right; S, sulcus; S, superior; sup, supramarginal; T, temporal; tr, transversal). Adapted from [98]. (F) Mean scaling exponents ( $\beta$ ) of long-range temporal correlations (LRTCS) in alpha-band oscillations during stimulus-detection task (Task) and in a separate resting-state 
recently, CFS has also been observed in source-reconstructed MEG/EEG data during parametric visual WM (VWM) tasks [98] (Figure 3E). There, WWM maintenance was characterized by CFS of theta with alpha, beta, and gamma oscillations as well as by CFS of high-alpha with beta and gamma oscillations. These data suggest that CFS is a functionally significant communication mechanism that, at least hypothetically, could underlie temporal segmentation and coordination of neuronal processing across multiple temporal scales.

\section{Scale-Free Behavioral and Neuronal Fluctuations}

Human cognitive performance is highly variable, but fluctuates in an autocorrelated manner so that performance in any given trial is similar to the preceding trials [99]. These autocorrelations are power-law distributed, long-range temporal correlations (LRTCs) that last up to hundreds of seconds (Figure 1F) [100-102]. Similar slow fluctuations and LRTCs also characterize the amplitude envelopes of $>1 \mathrm{~Hz}$ oscillations in electrophysiological data and blood-oxygen-level dependent (BOLD) signals in functional magnetic resonance imaging (fMRI) data [101, 103-105]. Moreover, neuronal and behavioral LRTCs are correlated so that subjects with strong LRTCs in neuronal fluctuations, both at rest and during the task, also have strong LRTCs in behavioral fluctuations [103] (Figure 3F,G), which shows that LRTCs reflect a trait-like dynamic of spontaneous brain activity that is preserved during task execution.

LRTCs in neuronal activity are reflected in slow and infraslow $(0.01-1 \mathrm{~Hz})$ fluctuations that are observable directly in slow EEG potentials, in addition to the amplitude envelopes and fMRI BOLD signals. These slow fluctuations are scale-free (i.e., have a power-law spectrum, but can either be composed of arrhythmic noise or quasiperiodic oscillations [Figure 1F,G]). Supporting the latter alternative, in scalp EEG [101], the phase but not the amplitude of the infraslow fluctuations predicts the detection of weak sensory stimuli so that slow fluctuations segment behavior into streaks of detected and undetected stimuli (Figure 1F). The slow EEG scalp potential fluctuations are direct electrophysiological correlates of the fMRI resting-state network activity [106] (Figure 3H) and also BOLD fluctuations are predictive of detection performance variability [107-109]. fMRI data suggest that the performance fluctuations may arise from the antagonistic interplay between the default mode and attentional or cingulo-opercular control networks $[107,110,111]$.

Both in scalp EEG [101] and fMRI, these slow scalp-potential (Figure 1F) and hemodynamic fluctuations are coupled via PAC with $1-40 \mathrm{~Hz}$ oscillations (Figure 3D). This suggests that similarly to delta, theta, and alpha oscillations, these slow fluctuations reflect excitability fluctuations in neuronal networks leading to temporal segmentation of neuronal processing, and consequently also behavioral performance, in the slow timescales.

\section{Brain Criticality as a Mechanism for Slow Fluctuations}

LRTCs and power-law scaling are a phenomenological signature of systems operating in or near a critical state $[4,112]$. Several lines of evidence have shown similar indications of critical dynamics in neuronal activity, including avalanche dynamics and LRTCs. Despite growing evidence that slow fluctuations and scale-free neuronal dynamics modulate behavioral

session (Rest) are correlated with the mean behavioral scaling exponents of task data ( $\left.\beta_{\text {behav }}\right)$. (G) Correlations between behavioral scaling exponents, $\beta$, and neuronal LRTCs in the resting state well delineated in specific brain areas. Adapted from [103]. (H) Infraslow fluctuations in full-band EEG (fbEEG) and fMRI BOLD signals exhibit correlations in the same anatomical regions. BOLD independent components (red-yellow) are overlaid by correlation maps between fbEEG independent components and $\mathrm{AMRI}$ voxel signals (green; VIS, visual network; DMN, default mode network; DAN, dorsal attention network; SAL, saliency network; S2, secondary somatosensory network; M1, primary motor network; EXEC, executive contro network). Adapted from [106]. 
performance, these phenomena have been largely thought to be intrinsic or even epiphenomenal properties of brain activity, with possibly no functional relationship to the dynamics of natural environments. Yet, critical brain dynamics has been proposed to be essential for enabling rapid switching between metastable states and to maximize information transfer and capacity of the system as well as its dynamic range [4,112]. Critical dynamics emerge in systems poised at a phase transition between two states, here termed the 'subcritical' and 'supercritical' states. At the critical point, spatiotemporally global correlation patterns, such as synchronization, can emerge from local interaction mechanisms. Brains, and in particular brain oscillations, exhibit critical-like dynamics $[4,105,112,113]$. Criticality endows the system with maximal information capacity, transmission capability [114], and dynamic range [115]. In particular, criticality has fundamental implications to functional properties of neuronal network oscillations, where it indicates a balance between disorder and order (i.e., between too low and excessively high synchronization). Despite such theoretical predictions, research fields exploring the functional roles of neuronal oscillations, as assessed here, have been only scarcely connected with the field of neuronal criticality [116]. At the critical point with intermediate mean synchronization, the long-range correlations and power-law scaling emerge in oscillatory dynamics, and the variability of synchronization is maximized [117,118] (Figure 4A,B). It should

(A)
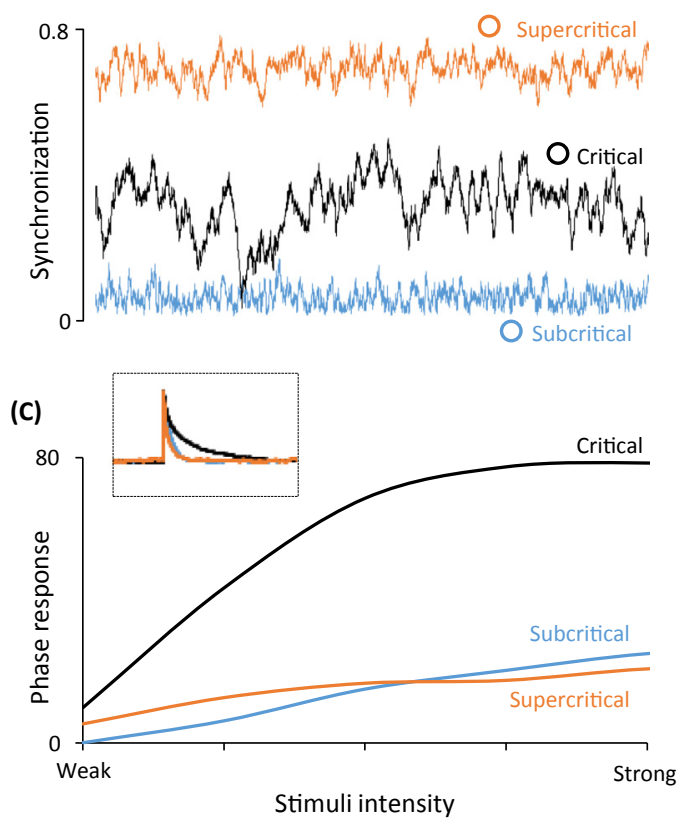

(B)

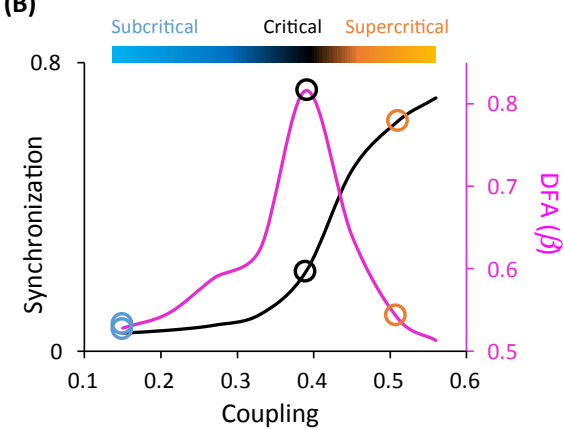

(D)

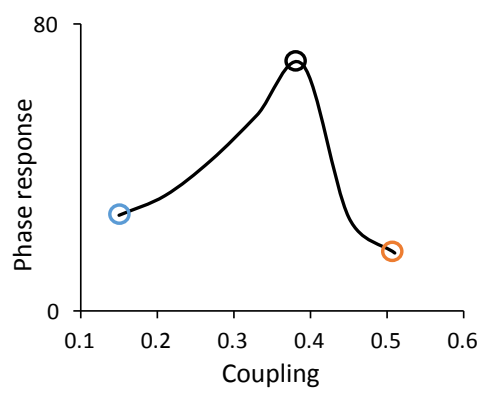

Figure 4. Critical Dynamics Endows Locally Coupled Oscillatory Systems Long-Range Spatiotempora Correlations and Maximizes Phase Reset Responses. (A) Simulated synchronization time series in a Kuramoto model, a network of phase coupled oscillators, poised at subcritical (blue), critical (black), and supercritical (orange) regimes. (B) The 'control parameter' in this model is strength of mean coupling among oscillators ( $x$-axis). Synchronization (black line), i.e., the 'order parameter', increases monotonically with increasing coupling. At the critical phase transition between disordered (low synchronization) and ordered (high synchronization), the system exhibits the greatest variability and exhibits emergent, long-range, power-law correlations, as indicated by the peaking of the scaling exponent, $\beta$, of detrended fluctuation analysis (DFA, magenta line). (C,D) In the critical state, the system exhibits more long-lasting responses to external phase-resetting stimuli than in subcritical or supercritical states, showing that changes in the system's operating point along the subcritical to supercritical axis may have significant consequences for phase resetting and entrainment in neuronal systems. 
be noted, however, that at the whole-brain scale, and unlike in classical theoretical models of criticality in homogenous systems, both neuronal synchronization and criticality are co-organized in modular structures [116]. This is in line with theoretical studies showing that in heterogeneous or, in particular, hierarchically modular and real brain networks, the critical point is 'stretched' into a wider critical regime [119]. Here the system operates in a 'Griffiths phase' where it exhibits 'critical-like', rather than strictly critical, dynamics.

We propose that critical dynamics of neuronal oscillations, despite being reflected in their slow fluctuations, are a prerequisite for fast and dynamic adaptation to environmental, natural, statistical regularities, and for the underlying capability for phase resetting and entrainment. If the neuronal system is set too far into the subcritical domain, neuronal oscillations are too weakly synchronized. In this case, neuronal oscillations will be unable to impose the intrinsic periodicity required for effective processing or to propagate the entrainment needed for synchronization with extrinsic signals. Conversely, in a system that is too far into supercritical settings, neuronal oscillations exhibit excessive synchronization. Here, information engrained in finer spatiotemporal structures is lost, and neural circuits are unable to flexibly alter their phase dynamics for entrainment. In sum, neuronal oscillations that are near criticality and at the optimal intermediate levels of synchronization are most flexible; they are able to achieve rapid transitions into novel states via phase-resetting (Figure 4C,D), and to adapt to the periodicities in sensory information. This is particularly important in the context of scale-free, possibly only quasiperiodic, environmental rhythmicities (Figure 1 and Box 1), where brain mechanisms must extract temporal information and adapt to the dynamically varying periodicity rapidly.

\section{Concluding Remarks and Future Perspectives}

The studies reviewed here show that across a wide range of timescales, brain dynamics can explicitly predict temporal intervals as well as adapt to behaviorally relevant environmental rhythmicities by phase prediction and entrainment of neuronal oscillations. They also show that even in the absence of explicit environmental, temporal information, endogenous neuronal oscillations impose rhythmicity on perceptual, attentional, and action-generation processes. Crucially, the intrinsic pacing of sensorimotor and cognitive functions via 1-15 Hz oscillations appears to be a central mechanism for parsing continuous sensory input streams and for achieving neuronal coordination in large-scale brain circuits. Although a number of studies have examined the relationship between brain oscillations and temporal predictions in simplified laboratory settings, fewer studies have addressed sensory sampling in more natural environments where incoming sensory information is scale-free and comprised of a variety of timescales. This exciting line of research suggests that the sampling of natural sensory information across multiple scales correspondingly involves neuronal oscillations in multiple frequency bands and necessitates CFC mechanisms. We propose that CFC enables coordinated cooperation between the oscillating assemblies and communication between them. Putatively, these coupling mechanisms encompass alpha-band perceptual/motor sampling, beta-band attentional sampling, and delta oscillations supporting functions such as active sensing and speech perception. In particular, while PAC readily couples these slow oscillations with gamma-band activities, it has remained less clear how the spectrally nearby $1-15 \mathrm{~Hz}$ oscillations are mutually coordinated. We propose that one possible mechanism could be CFS that flexibly couples oscillations with small (e.g., 1:2 and 1:3) frequency ratios with the temporal accuracy of the faster oscillation and hence parse multiscale sensorimotor information and cognitive operations. Future studies are required to elucidate the mechanistic roles of PAC and CFS in cross-scale coordination of neuronal processing.

\section{Outstanding Questions}

The prestimulus phase can bias behavioral outcomes. What are the brain structures implicated in this process? Does the bias arise in sensory or attentional systems, and how is the localization dependent on the relevant oscillation frequency?

Large-scale networks of interareal phase coupling coordinate attention and conscious perception. Are the prestimulus phase biases associated with the hubs of these networks and are they related to specific interareal phase relationships?

What is the role of thalamocortical alpha-band rhythmicity in perceptual sampling?

Are temporal predictions arising from oscillatory processes at multiple frequencies coordinated via cross-frequency interactions? Does crossfrequency coupling play an instrumental role in this context or is it more of an epiphenomenon?

Do critical brain dynamics enable, or conversely limit, phase-locking and entrainment of neuronal activity according to sensorimotor demands?

Are infraslow fluctuations entrainable or adaptive, and, if so, do they contribute to sensorimotor predictions in the timescales of seconds to tens of seconds?

What is the mechanistic relationship between explicit time estimation and implicit temporal predictions? 
The functional significance of scale-free neuronal activity and behavioral performance fluctuations below $1 \mathrm{~Hz}$ remain another focal topic for future research. These slow fluctuations are observed in the fMRI BOLD signal as well as in the amplitude envelopes of fast $>1 \mathrm{~Hz}$ oscillations and $<1 \mathrm{~Hz}$ slow cortical potentials in electrophysiological data. These fluctuations are likely to be caused by emergent critical-like brain dynamics. In the context of temporal expectations, the dynamic state of the neuronal systems at hand (whether critical or subcritical/ supercritical) is rarely considered. The dynamic state could contribute significantly to the ability of neuronal oscillations to express flexible synchronization, phase resetting, and entrainment. In addition, it is tempting to speculate that the dynamic state of neural systems, apart from its variation across specific brain systems and different external conditions, also varies between individuals, possibly through heritable processes and may thus contribute to the interindividual variability in corresponding perceptual and attentional measures (see Outstanding Questions).

\section{Acknowledgements}

This work was funded by the Academy of Finland. We are grateful to Hamed Haque and Sami Karadeniz for the illustration in Figure 2E and the corresponding Table S1 in the supplemental information online, and to Sheng Wang for the schematic simulations for Figure 4.

\section{Supplemental Information}

Supplemental information associated with this article can be found online at https://doi.org/10.1016/j.tins.2018.08.008.

\section{References}

1. van der Schaaf, A. and van Hateren, J.H. (1996) Modelling the power spectra of natural images: statistics and information. Vis. Res. 36, 2759-2770

2. Singh, N.C. and Theunissen, F.E. (2003) Modulation spectra of natural sounds and ethological theories of auditory processing. J. Acoust. Soc. Am. 114, 3394-3411

3. Shew, W.L. and Plenz, D. (2012) The functional benefits of criticality in the cortex. Neuroscientist 19, 88-100

4. Chialvo, D.R. (2010) Emergent complex neural dynamics. Nat. Phys. 6, 744-750

5. Fries, P. (2015) Rhythms for cognition: communication through coherence. Neuron 88, 220-235

6. Herrmann, B. et al. (2016) Temporal expectations and neural amplitude fluctuations in auditory cortex interactively influence perception. Neuroimage 124, 487-497

7. Besle, J. et al. (2011) Tuning of the human neocortex to the temporal dynamics of attended events. J. Neurosci. 31, 31763185

8. Stefanics, G. et al. (2010) Phase entrainment of human delta oscillations can mediate the effects of expectation on reaction speed. J. Neurosci. 30, 13578-13585

9. Lakatos, P. et al. (2008) Entrainment of neuronal oscillations as a mechanism of attentional selection. Science 320, 110-113

10. Gong, G. et al. (2009) Mapping anatomical connectivity patterns of human cerebral cortex using in vivo diffusion tensor imaging tractography. Cereb. Cortex 19, 524-536

11. Ding, N. et al. (2017) Temporal modulations in speech and music. Neurosci. Biobehav. Rev. 81, 181-187

12. Arnal, L.H. et al. (2015) Delta-beta coupled oscillations underlie temporal prediction accuracy. Cereb. Cortex 25, 3077-3085

13. Teng, $X$. et al. (2017) Concurrent temporal channels for auditon processing: oscillatory neural entrainment reveals segregation of function at different scales. PLoS Biol. 15, e2000812

14. Keitel, A. et al. (2018) Perceptually relevant speech tracking in auditory and motor cortex reflects distinct linguistic features. PLoS Biol. 16, e2004473

15. Ding, N. et al. (2016) Cortical tracking of hierarchical linguistic structures in connected speech. Nat. Neurosci. 19, 158-164

16. Wilsch, A. et al. (2018) Transcranial alternating current stimulation with speech envelopes modulates speech comprehension. Neuroimage 172, 766-774

17. Keitel, C. et al. (2017) Visual cortex responses reflect temporal structure of continuous quasi-rhythmic sensory stimulation. Neuroimage 146, 58-70

18. Montemurro, M.A. et al. (2008) Phase-of-firing coding of natural visual stimuli in primary visual cortex. Curr. Biol. 18, 375-380

19. VanRullen, R. (2016) Perceptual cycles. Trends Cogn. Sci. 20 723-735

20. Haegens, S. et al. (2011) Beta oscillations in the monkey sensorimotor network reflect somatosensory decision making. Proc. Natl. Acad. Sci. U. S. A. 108, 10708-10713

21. Dugue, L. et al. (2011) The phase of ongoing oscillations mediates the causal relation between brain excitation and visual perception. J. Neurosci. 31, 11889-11893

22. Drewes, J. and Vanrullen, R. (2011) This is the rhythm of you eyes: the phase of ongoing electroencephalogram oscillations modulates saccadic reaction time. J. Neurosci. 31, 4698 4708

23. Busch, N.A. and VanRullen, R. (2010) Spontaneous EEG oscillations reveal periodic sampling of visual attention. Proc. Natl. Acad. Sci. U. S. A. 107, 16048-16053

24. Mathewson, K.E. et al. (2009) To see or not to see: prestimulus alpha phase predicts visual awareness. J. Neurosci. 29, 27252732

25. Hanslmayr, S. et al. (2013) Prestimulus oscillatory phase at $7 \mathrm{~Hz}$ gates cortical information flow and visual perception. Curr. Biol. 23, 2273-2278

26. Sherman, M.T. et al. (2016) Rhythmic influence of top-down perceptual priors in the phase of prestimulus occipital alpha oscillations. J. Cogn. Neurosci. 28, 1318-1330

27. Samaha, J. and Postle, B.R. (2015) The speed of alpha-band oscillations predicts the temporal resolution of visual perception. Curr. Biol. 25, 2985-2990

28. Sokoliuk, R. and VanRullen, R. (2013) The flickering wheel illusion: when alpha rhythms make a static wheel flicker. J. Neurosci. 33, 13498-13504 
29. VanRullen, R. and Macdonald, J.S. (2012) Perceptual echoes at $10 \mathrm{~Hz}$ in the human brain. Curr. Biol. 22, 995-999

30. VanRullen, R. et al. (2005) Attention-driven discrete sampling of motion perception. Proc. Natl. Acad. Sci. U. S. A. 102, 52915296

31. VanRullen, R. et al. (2007) The blinking spotlight of attention. Proc. Natl. Acad. Sci. U. S. A. 104, 19204-19209

32. Landau, A.N. et al. (2015) Distributed attention is implemented through theta-rhythmic gamma modulation. Curr. Biol. 25, 2332-2337

33. Landau, A.N. and Fries, P. (2012) Attention samples stimuli rhythmically. Curr. Biol. 22, 1000-1004

34. Fiebelkorn, I.C. et al. (2013) Rhythmic sampling within and between objects despite sustained attention at a cued location. Curr. Biol. 23, 2553-2558

35. Samaha, J. et al. (2015) Top-down control of the phase of alpha-band oscillations as a mechanism for temporal prediction. Proc. Natl. Acad. Sci. U. S. A. 112, 8439-8444

36. Lobier, M. et al. (2017) High-alpha band synchronization across frontal, parietal and visual cortex mediates behavioral and neuronal effects of visuospatial attention. Neuroimage 165, 222-237

37. Ai, L. and Ro, T. (2014) The phase of prestimulus alpha oscillations affects tactile perception. J. Neurophysiol. 111, 1300-1307

38. Baumgarten, T.J. et al. (2015) Beta oscillations define discrete perceptual cycles in the somatosensory domain. Proc. Natl. Acad. Sci. U. S. A. 112, 12187-12192

39. Ng, B.S. et al. (2012) A precluding but not ensuring role of entrained low-frequency oscillations for auditory perception. J. Neurosci. 32, 12268-12276

40. Strauss, A. et al. (2015) Alpha phase determines successful lexical decision in noise. J. Neurosci. 35, 3256-3262

41. Zoefel, B. and VanRullen, R. (2017) Oscillatory mechanisms of stimulus processing and selection in the visual and auditory systems: state-of-the-art, speculations and suggestions. Front. Neurosci. 11, 296

42. Dugue, L. et al. (2011) The phase of ongoing oscillations mediates the causal relation between brain excitation and visual perception. J. Neurosci. 31, 11889-11893

43. Emrich, S.M. et al. (2017) Comparing the effects of $10-\mathrm{Hz}$ repetitive TMS on tasks of visual STM and attention. J. Cogn. Neurosci. 29, 286-297

44. Neuling, T. et al. (2012) Good vibrations: oscillatory phase shapes perception. Neuroimage 63, 771-778

45. Melloni, L. et al. (2009) (Micro)saccades, corollary activity and cortical oscillations. Trends Cogn. Sci. 13, 239-245

46. Bosman, C.A. et al. (2009) A microsaccadic rhythm modulates gamma-band synchronization and behavior. J. Neurosci. 29, 9471-9480

47. Chen, C.Y. et al. (2015) Neuronal response gain enhancement prior to microsaccades. Curr. Biol. 25, 2065-2074

48. Amit, R. et al. (2017) Temporal dynamics of saccades explained by a self-paced process. Sci. Rep. 7, 886

49. Haegens, S. et al. (2011) Alpha-oscillations in the monkey sensorimotor network influence discrimination performance by rhythmical inhibition of neuronal spiking. Proc. Natl. Acad. Sci. U. S. A. 108, 19377-19382

50. Bollimunta, A. et al. (2008) Neuronal mechanisms of cortical alpha oscillations in awake-behaving macaques. J. Neurosci. 28, 9976-9988

51. Maldonado, P. et al. (2008) Synchronization of neuronal responses in primary visual cortex of monkeys viewing natural images. J. Neurophysiol. 100, 1523-1532

52. Rajkai, C. et al. (2008) Transient cortical excitation at the onset of visual fixation. Cereb. Cortex 18, 200-209

53. Neupane, S. et al. (2017) Coherent alpha oscillations link current and future receptive fields during saccades. Proc. Natl. Acad. Sci. U. S. A. 114, E5979-E5985
54. Hoffman, K.L. et al. (2013) Saccades during visual exploration align hippocampal $3-8 \mathrm{~Hz}$ rhythms in human and non-human primates. Front. Syst. Neurosci. 7, 43

55. Bollimunta, A. et al. (2011) Neuronal mechanisms and attentional modulation of corticothalamic alpha oscillations. $J$ Neurosci. 31, 4935-4943

56. Haegens, S. et al. (2015) Laminar profile and physiology of the alpha rhythm in primary visual, auditory, and somatosensory regions of neocortex. J. Neurosci. 35, 14341-14352

57. Peel, T.R. et al. (2016) A causal role for the cortical frontal eye fields in microsaccade deployment. PLoS Biol. 14, e1002531

58. Buschman, T.J. and Miller, E.K. (2007) Top-down versus bottom-up control of attention in the prefrontal and posterior parietal cortices. Science 315, 1860-1862

59. Gregoriou, G.G. et al. (2012) Cell-type-specific synchronization of neural activity in FEF with V4 during attention. Neuron 73, $581-594$

60. Saalmann, Y.B. et al. (2012) The pulvinar regulates information transmission between cortical areas based on attention demands. Science 337, 753-756

61. Neggers, S.F. et al. (2007) TMS pulses on the frontal eye fields break coupling between visuospatial attention and eye movements. J. Neurophysiol. 98, 2765-2778

62. Marshall, T.R. et al. (2015) Frontal eye fields control attentiona modulation of alpha and gamma oscillations in contralatera occipitoparietal cortex. J. Neurosci. 35, 1638-1647

63. Hamel-Thibault, A et al. (2018) Delta-band oscillations in moto regions predict hand selection for reaching. Cereb. Cortex 28 $574-584$

64. Saleh, M. et al. (2010) Fast and slow oscillations in human primary motor cortex predict oncoming behaviorally relevant cues. Neuron 65, 461-471

65. Gross, J. et al. (2002) The neural basis of intermittent moto control in humans. Proc. Natl. Acad. Sci. U. S. A. 99, 22992302

66. Salenius, S. and Hari, R. (2003) Synchronous cortical oscillaton activity during motor action. Curr. Opin. Neurobiol. 13, 678-684

67. Schoffelen, J.M. et al. (2011) Selective movement preparation is subserved by selective increases in corticomuscular gammaband coherence. J. Neurosci. 31, 6750-6758

68. Bourguignon, M. (2017) MEG insight into the spectral dynamics underlying steady isometric muscle contraction. J. Neurosci. 37 , 10421-10437

69. Witham, C.L. et al. (2010) Corticomuscular coherence between motor cortex, somatosensory areas and forearm muscles in the monkey. Front. Syst. Neurosci. 4, 38

70. Mackay, W.A. (1997) Synchronized neuronal oscillations and their role in motor processes. Trends Cogn. Sci. 1, 176-183

71. Hirvonen, J. et al. (2018) Dynamic large-scale network synchronization from perception to action. Netw. Neurosci. Published online January 10, 2018. http://dx.doi.org/10.1162/netn_a 00039

72. Assaneo, M.F. and Poeppel, D. (2018) The coupling between auditory and motor cortices is rate-restricted: evidence for an intrinsic speech-motor rhythm. Sci. Adv. 4, eaao3842

73. Bergmann, T.O. et al. (2012) EEG-guided transcranial magnetic stimulation reveals rapid shifts in motor cortical excitability during the human sleep slow oscillation. J. Neurosci. 32, 243-253

74. van Elswijk, G. et al. (2010) Corticospinal beta-band synchronization entails rhythmic gain modulation. J. Neurosci. 30, 44814488

75. Merchant, H. et al. (2013) Neural basis of the perception and estimation of time. Annu. Rev. Neurosci. 36, 313-336

76. Treisman, M. et al. (1990) The internal clock: evidence for a temporal oscillator underlying time perception with some estimates of its characteristic frequency. Perception 19, 705-743

77. Matell, M.S. and Meck, W.H. (2004) Cortico-striatal circuits and interval timing: coincidence detection of oscillatory processes. Brain Res. Cogn. Brain Res. 21, 139-170 
78. Buhusi, C.V. and Meck, W.H. (2009) Relativity theory and time perception: single or multiple clocks? PLoS One 4, e6268

79. van Rijn, H. et al. (2011) Contingent negative variation and its relation to time estimation: a theoretical evaluation. Front. Integr. Neurosci. 5, 91

80. Kosem, A. et al. (2014) Encoding of event timing in the phase of neural oscillations. Neuroimage 92, 274-284

81. Bartolo, R. and Merchant, H. (2015) Beta oscillations are linked to the initiation of sensory-cued movement sequences and the internal guidance of regular tapping in the monkey. J. Neurosci. 35, 4635-4640

82. Kulashekhar, S. et al. (2016) The role of cortical beta oscillations in time estimation. Hum. Brain Mapp. 37, 3262-3281

83. Palva, J.M. et al. (2005) Phase synchrony among neuronal oscillations in the human cortex. J. Neurosci. 25, 3962-3972

84. Palva, J.M. and Palva, S. (2017) Functional integration across oscillation frequencies by cross-frequency phase synchronization. Eur. J. Neurosci. Published online November 2, 2017. http://dx.doi.org/10.1111/ejn.13767

85. Lakatos, P. et al. (2005) An oscillatory hierarchy controlling neuronal excitability and stimulus processing in the auditory cortex. J. Neurophysiol. 94, 1904-1911

86. Jensen, O. et al. (2014) Temporal coding organized by coupled alpha and gamma oscillations prioritize visual processing. Trends Neurosci. 37, 357-369

87. Sporns, O. and Kotter, R. (2004) Motifs in brain networks. PLoS Biol. 2, e369

88. Watrous, A.J. et al. (2015) Phase-amplitude coupling supports phase coding in human ECoG. Elife 4, e07886

89. Honkanen, R. et al. (2015) Gamma oscillations underlie the maintenance of feature-specific information and the contents of visual working memory. Cereb. Cortex 25, 3788-3801

90. Lakatos, P. et al. (2016) Global dynamics of selective attention and its lapses in primary auditory cortex. Nat. Neurosci. 19, $1707-1717$

91. Axmacher, N. et al. (2010) Cross-frequency coupling supports multi-item working memory in the human hippocampus. Proc. Natl. Acad. Sci. U. S. A. 107, 3228-3233

92. Gross, J. et al. (2013) Speech rhythms and multiplexed oscillatory sensory coding in the human brain. PLoS Biol. 11, e1001752

93. Giraud, A.L. and Poeppel, D. (2012) Cortical oscillations and speech processing: emerging computational principles and operations. Nat. Neurosci. 15, 511-517

94. Tass, P. et al. (1998) Detection of $n: m$ phase locking from noisy data: application to magnetoencephalography. Phys. Rev. Lett. 81, 3291-3294

95. Fell, J. and Axmacher, N. (2011) The role of phase synchronization in memory processes. Nat. Rev. Neurosci. 12, 105-118

96. Akiyama, M. et al. (2017) Theta-alpha EEG phase distributions in the frontal area for dissociation of visual and auditory working memory. Sci. Rep. 7, 42776

97. Sauseng, P. et al. (2008) Cross-frequency phase synchronization: a brain mechanism of memory matching and attention. Neuroimage 40, 308-317

98. Siebenhühner, F. et al. (2016) Cross-frequency synchronization connects networks of fast and slow oscillations during visual working memory maintenance. Elife 5, e13451

99. Verplanck, W.S. et al. (1952) Nonindependence of successive responses in measurements of the visual threshold. J. Exp. Psychol. 44, 273-282
100. Gilden, D.L. and Wilson, S.G. (1995) On the nature of streaks in signal detection. Cognit. Psychol. 28, 17-64

101. Monto, S. et al. (2008) Very slow EEG fluctuations predict the dynamics of stimulus detection and oscillation amplitudes in humans. J. Neurosci. 28, 8268-8272

102. Simola, J. et al. (2017) Critical dynamics of endogenous fluctuations predict cognitive flexibility in the Go/NoGo task. Sci. Rep. 7, 2909-017-02750-9

103. Palva, J.M. et al. (2013) Neuronal long-range temporal correlations and avalanche dynamics are correlated with behaviora scaling laws. Proc. Natl. Acad. Sci. U. S. A. 110, 3585-3590

104. He, B.J. (2011) Scale-free properties of the functional magnetic resonance imaging signal during rest and task. J. Neurosci. 31 13786-13795

105. Linkenkaer-Hansen, K. et al. (2001) Long-range tempora correlations and scaling behavior in human brain oscillations. J. Neurosci. 21, 1370-1377

106. Hiltunen, T. et al. (2014) Infra-slow EEG fluctuations are corre lated with resting-state network dynamics in fMRI. J. Neurosci. 34, 356-362

107. Boly, M. et al. (2007) Baseline brain activity fluctuations predict somatosensory perception in humans. Proc. Natl. Acad. Sci. U. S. A. 104, 12187-12192

108. Sadaghiani, S. et al. (2009) Distributed and antagonistic contributions of ongoing activity fluctuations to auditory stimulus detection. J. Neurosci. 29, 13410-13417

109. Spadone, S. et al. (2015) Dynamic reorganization of human resting-state networks during visuospatial attention. Proc. Nat. Acad. Sci. U. S. A. 112, 8112-8117

110. Fox, M.D. et al. (2005) The human brain is intrinsically organized into dynamic, anticorrelated functional networks. Proc. Natl. Acad. Sci. U. S. A. 102, 9673-9678

111. Sadaghiani, S. et al. (2010) The relation of ongoing brain activity, evoked neural responses, and cognition. Front. Syst. Neurosci. 4,20

112. Cocchi, L. et al. (2017) Criticality in the brain: a synthesis of neurobiology, models and cognition. Prog. Neurobiol. 158, 132 152

113. Beggs, J.M. and Plenz, D. (2003) Neuronal avalanches in neocortical circuits. J. Neurosci. 23, 11167-11177

114. Shew, W.L. et al. (2011) Information capacity and transmission are maximized in balanced cortical networks with neurona avalanches. J. Neurosci. 31, 55-63

115. Shew, W.L. et al. (2009) Neuronal avalanches imply maximum dynamic range in cortical networks at criticality. J. Neurosci. 29, 15595-15600

116. Zhigalov, A. et al. (2017) Modular co-organization of functiona connectivity and scale-free dynamics in the human brain. Netw. Neurosci. 1, 143-165

117. Yang, H. et al. (2012) Maximal variability of phase synchrony in cortical networks with neuronal avalanches. J. Neurosci. 32, 1061-1072

118. Poil, S.S. et al. (2012) Critical-state dynamics of avalanches and oscillations jointly emerge from balanced excitation/inhibition in neuronal networks. J. Neurosci. 32, 9817-9823

119. Moretti, P. and Munoz, M.A. (2013) Griffiths phases and the stretching of criticality in brain networks. Nat. Commun. 4, 2521

120. Vanhatalo, S. et al. (2004) Infraslow oscillations modulate excitability and interictal epileptic activity in the human cortex during sleep. Proc. Natl. Acad. Sci. U. S. A. 101, 5053-5057 